\title{
Editorial
}

\section{Women's Health: Infertility- Needs a Team Approach of Both Physician \& Gynecologist}

Women's health has an intimate connection to the health of children and families. ${ }^{1}$ Women's health has become a distinct clinical discipline with a focus on disorders that occur disproportionately in women. The integration of women's health into internal medicine and other specialties has been accompanied by novel approaches to health care delivery, including greater attention to patient education and involvement in disease prevention and medical decision. The goals of improving women's health have been in place and recognized for some time from the first International Safe Motherhood Conference in 1987 to the International Conference for Population and Development (ICPD) in 1994, the Fourth World Conference on Women in 1995, and ICPD +5 in $1999 .^{2}$

The WHO defines infertility as 'Infertility is the inability to conceive a child. A couple may be considered infertile if, after two years of regular sexual intercourse, without contraception, the woman has not become pregnant (and there is no other reason, such as breastfeeding or postpartum amenorrhea.

Infertility remains an anonymous epidemic, with less funding and research than other common medical problems receive. Some estimates suggest that worldwide "between three and seven per cent of all couples or women have an unresolved problem of infertility.It is believed that the presence of children in a marriage ensures that the household name is sustained and the living touch of human existence is not extinguished. ${ }^{3,4}$ Childlessness thus attracts stigma and profound negative consequences especially for the women folk. This is particularly true of most developing countries where a woman's status is often defined by her reproductive capacity and womanhood is equated with motherhood. ${ }^{5}$ Reported consequences of infertility include psychological, social and physical suffering. It affects the health and wellbeing of the individuals concerned as well as society. Anxiety, depression, lowered life satisfaction, frustration, grief, fear, guilt, helplessness, reduced job performance, marital duress, dissolution and abandonment economic hardship, loss of social status, social stigma, social isolation and alienation, community ostracism, physical violence and sometimes painful and life threatening medical intervention are documented correlates of childlessness particularly in developing countries. ${ }^{6}$
A number of studies have found that the incidence of depression in infertile couples presenting for infertility treatment is significantly higher than in fertile controls, with prevalence estimates of major depression in the range of $15 \%-54 \% .^{7}$ Anxiety has also been shown to be significantly higher in infertile couples when compared to the general population, with $8 \%-28 \%$ of infertile couples reporting clinically significant anxiety. ${ }^{8}$ The causal role of psychological disturbances in the development of infertility is still a matter of debate.

According to the American Society for Reproductive Medicine (ASRM), age, smoking, sexually transmitted infections, and being overweight or underweight, endocrine diseases (hypopituitarism congenital adrenal hyperplasia virilizing tumors adrenal failure cushing syndrome hypo/ hyperthyroidism, diabetes mellitus) genetic factors and psychological disturbance can all affect fertility. ${ }^{9}$

In 1957 one large study found that by age 35, 11\% of couples were infertile, by age $40,33 \%$ of couples were infertile, at age $45,87 \%$ of couples were infertile. Smokers are $60 \%$ more likely to be infertile than non-smokers. Smoking reduces the chances of IVF producing a live birth by $34 \%$ and increases the risk of an IVF pregnancy miscarrying by $30 \%$. Also, female smokers have an earlier onset of menopause by approximately $1-4$ years. Twelve percent of all infertility cases are a result of a woman either being underweight or overweight. A study in the US indicated that approximately $20 \%$ of infertile women had a past or current eating disorder, which is five times higher than the general lifetime prevalence rate. Chemotherapy poses a high risk of infertility. Other factors that can cause acquired infertility are significant liver or kidney disease. Thrombophilia, cannabis smoking, such as of marijuana causes disturbances in the end cannabinoid system, potentially causing infertility. Environmental factors -toxins such as glues, volatile organic solvents or silicones, physical agents, chemical dusts, and pesticides. Specific female causes of infertility include: ovulation problems (e.g. polycystic ovarian syndrome) tubal blockage, pelvic inflammatory disease, uterine problems, previous tubal ligation, endometriosis and advanced maternal age. ${ }^{9}$ 
Treatment depends on the cause of infertility, but may include counseling, healthy lifestyle advice, treatment of medical conditions associated with infertility, fertility treatments, which include ovulation induction with antiestrogen, clomiphene citrate and assisted reproduction \&psychotherapy.

The physician's role in preparing the patient for pregnancy has become more prominent as more women with medical illness are able to bear children. It is estimated that $15 \%$ of all women beginning prenatal care have a medical illness. Their role are extremely important in preconception interventions for medical conditions associated with infertility, evaluation of patients before treatment with assisted reproductive technology \& in dealing with direct and indirect complications of infertility treatments. Patients seeking assisted reproductive technology are likely to be older and to have concomitant medical conditions, which may have originally contributed to the infertility. Other underlying medical illnesses, such as thrombophilia, may be exacerbated or unmasked by the complications of the drugs used for assisted reproductive technology.

All women should be counseled on the benefits of smoking cessation and the importance of folic acid for reduction of neural tube defects. They should also be screened for rubella and questioned about family history of genetic disorders and thrombophilia. Diabetic women should be told of the importance of normalizing hemoglobin A1c levels before conception to prevent congenital anomalies. Preconception counseling should address the potential effects of both the assisted reproductive technology and its success. The effects of assisted reproductive technology and pregnancy on any underlying medical illness should be discussed.

Internists can and should contribute to the care of all medically compromised pregnant women. The physician is also likely to see the patient before pregnancy or infertility interventions begin. A full knowledge of the effects of such interventions on medical illness and the impact of medical conditions on pregnancy will help clinicians counsel women considering assisted reproductive technology and will provide an opportunity for early intervention. Several studies suggest that cognitive behavioral group psychotherapy and support groups decrease stress and mood symptoms, as well as increase fertility rates.
Infertility treatment isn't always successful. And that's the problem with how infertility is being handled; as with any other disease, some people won't be cured. That's why it needs more recognition and involvement of different disciplines for comprehensive management.

So the success of treatment depends on active participation $\&$ collaboration of Physician, Reproductive endocrinologists, Gynecologist, Psychiatrist and social support group.

19 th August

\section{Professor MA Kashem Khondokar}

Prof. of Medicine

Vice Principal, Popular Medical College

\& Former President, Bangladesh Society of Medicine

\section{References:}

1. Tinker A, Finn,K, Joanne E. Improving Women's Health Issues and Interventions Health, Nutrition and Population (HNP) Discussion Paper, june, 2010

2. Usha R, SalganicoM.S. Kaiser Family Foundation Women's Health Care ChartbookKey Findings from theKaiser Women's Health SurveyMAY 2011

3. Mabasa, L.F. Stigma, Community Support and Therapy Methods of Infertility in South Africa: a Cultural Perspective.

4. Mbiti, J.S. Introduction to African Religion. Nairobi: Sunlitho Limited

5. Osagie G, Social and ethical aspects of assisted conception in Anglophone Sub-Saharan Africa, Report of a meeting on "Medical, ethical and social aspects of assisted reproduction. 17-21 September Geneva,Switzerland,WHO; 2001 p.50-54

6. Balen $\mathrm{V}$, Interpreting infertility a view from the social sciences). London.University of California Press

7. Domar, A.D., Zuttermeister, P.C. \& Friedman, R. The psychological impact of infertility: a comparison with patient with other medical conditions. Journal ofPsychosomatic Obstetrics and Gynaecology, 14 suppl; 45- 52

8. Awaritefe, A.TheAwaritefe Psychological Index. Nigerian Journal of Clinical Psychology, 1 (2): 42-51

9. Smals AGH, Kloppenborg PWC, Benraad TJ. Plasma testosterone profiles in Cushing's syndrome. J Clin Endocrinol Metab 1977; 45:240

10. Harrison's Online, Chapter 6. Women's Health 